\title{
Interval Robust Multi-objective Algorithm
}

\author{
G.L. Soares ${ }^{\text {b,c,a }}$, R.O. Parreiras ${ }^{\text {b,* }}$, L. Jaulin ${ }^{\text {c }}$, J.A. Vasconcelos ${ }^{\text {a }}$, C.A. Maia ${ }^{\text {a }}$ \\ ${ }^{\text {a } U n i v e r s i d a d e ~ F e d e r a l ~ d e ~ M i n a s ~ G e r a i s ~(U F M G), ~ B e l o ~ H o r i z o n t e, ~ B r a z i l ~}$ \\ ${ }^{\mathrm{b}}$ Pontifícia Universidade Católica de Minas Gerais (PUCMinas), Belo Horizonte, Brazil \\ c École Nationale Supérieure des Ingénieurs des Etudes des Techniques d'Armement (ENSIETA), Brest, France
}

\section{A R T I C L E I N F O}

\section{Keywords:}

Robust Multi-objective optimization

Robust Pareto frontier

Interval Analysis

Genetic algorithm

\begin{abstract}
A B S T R A C T
This paper introduces a method for solving multi-objective optimization problems in uncertain environment. When the uncertainty factors of the optimization problem can be included into the mathematical model, through bounded intervals, [I]RMOA (Interval Robust Multi-objective Algorithm) can find an enclosure of the robust Pareto frontier. In this approach, the robust Pareto solutions are the ones that have the best performance when the worst case scenario, characterized by the uncertainty parameters, is considered.

[I]RMOA has some positive aspects: it does not require the calculus of derivatives; it has only two input parameters; it is a reliable tool for solving different robust optimization problems, which can be nonlinear and discontinuous or have nonconvex Pareto frontier, for instance. The success of the method depends only on the quality of the objective inclusion functions and the precision parameters. Its disadvantage lies in the fact that it requires high computational effort, when high-dimensional problems are considered or when a very accurate enclosure is needed. Two analytical robust test functions are proposed to be treated by [I]RMOA and to validate the results provided by a stochastic multi-objective optimization method. The results are satisfactory.
\end{abstract}

(C) 2009 Elsevier Ltd. All rights reserved.

\section{Introduction}

The process of modeling the real system to be optimized involves several sources of uncertainties [1-3]. For instance, precision can be lost when constructing a computational model of the optimization parameters; the environment (factors such as humidity, pressure, and temperature) may produce effects on the real system, that are difficult to be quantified; there may be measurement imprecision in the estimative of the objective functions and of the optimization parameters. A possible approach for dealing with uncertainty factors consists in constructing an optimization model, which includes additional parameters that reflect the uncertainty effects [4-6]. The use of such an approach is interesting, as it allows one to adequately consider the uncertainty factors, since the beginning of the problem analysis. Stochastic and deterministic frameworks have been applied to treat optimization problems under uncertainties, such as $[7,8]$ for stochastic methods, and $[4,9]$ for deterministic ones.

Intervals have been applied to compute the uncertainties as in [10]. In a different approach, this paper proposes [I]RMOA (Interval Robust Multi-objective Algorithm), which utilizes concepts from the Interval Analysis to compute the uncertainty parameters during the search for an enclosure for the robust Pareto solutions. Here, as in [11,9,7], the robust Pareto solutions are the ones that have the best performance (reflected by the objective function evaluations), when the worst case scenario, characterized by the uncertainty parameters, is considered. The proposed algorithm has some advantageous properties, such

\footnotetext{
* Corresponding author.

E-mail addresses: gsoares@cpdee.ufmg.br (G.L. Soares), roberta.parreiras@terra.com.br (R.O. Parreiras), jaulinlu@ensieta.fr (L. Jaulin), joao@cpdee.ufmg.br (J.A. Vasconcelos), maia@cpdee.ufmg.br (C.A. Maia).
} 
as: it does not require the calculus of derivatives; it can be considered a versatile tool, as it always provides an enclosure of the robust Pareto frontier, even when complex problems or objective functions with different characteristics are considered (they can be nonlinear or linear, continuous or discontinuous, or it can have convex or nonconvex Pareto Frontier); it requires only two input parameters, whose effects in the results are very clear (both are associated with the accuracy of the enclosure). Besides, it is a reliable tool for solving robust optimization problems, when it is crucial to guarantee, that the real system will not operate under risk situations. We believe that [I]RMOA represents an important contribution, since incoming real applications which involve risk-averse strategies [12] are becoming frequent. However, its disadvantage lies in the fact that it requires high computational effort for solving high-dimensional problems or to generate very accurate enclosures.

This work introduces the [I]RMOA and the background on Multi-objective Optimization and Interval Analysis necessary to understand how it works. In order to evaluate the proposed algorithm, it is utilized for solving two analytical problems, with distinct characteristics. Besides, [I]RMOA is utilized to validate the Pareto frontier provided by an evolutionary algorithm. The results confirm the convenience of using the proposed methodology.

\section{Background}

\subsection{Multi-objective optimization}

Considering the design variables $\mathbf{x} \in \mathbf{X} \subseteq \mathbb{R}^{n}$ and the vector of objective functions $\boldsymbol{\Phi}(\mathbf{x}): \mathbb{R}^{n} \rightarrow \mathbb{R}^{m}$, the unconstrained minimization problem can be stated as:

$$
\min _{\mathbf{x} \in \mathbf{X}} \Phi(\mathbf{x}) \text {. }
$$

Two punctual solutions $\mathbf{u}$ and $\mathbf{v}$ from $\mathbf{X}$ can be compared on the basis of the order relation $\preceq$ (dominance relation), which is defined as:

$$
\boldsymbol{\Phi}(\mathbf{u}) \preceq \Phi(\mathbf{v}) \Leftrightarrow \Phi(\mathbf{u}) \leq \boldsymbol{\Phi}(\mathbf{v}), \quad \boldsymbol{\Phi}(\mathbf{u}) \neq \Phi(\mathbf{v}) .
$$

With the use of the dominance relation, it is possible to rank the solutions from best to worst. In the minimization case, the best solutions are the ones associated with the lowest objective function values $[13,14]$. Hence, solving (1) consists in finding the set:

$$
X^{*}=\left\{\mathbf{x}^{*} \in \mathbf{X} \mid \nexists \mathbf{x} \in \mathbf{X}, \boldsymbol{\Phi}(\mathbf{x}) \preceq \boldsymbol{\Phi}\left(\mathbf{x}^{*}\right)\right\} .
$$

If $\mathbf{Y}=\boldsymbol{\Phi}(\boldsymbol{X})$, then the Pareto frontier (or, equivalently, the set of non-dominated or efficient solutions) is:

$$
y^{*}=\left\{\mathbf{y}^{*} \in \mathbf{Y} \mid \nexists \mathbf{y} \in \mathbf{Y}, \mathbf{y} \preceq \mathbf{y}^{*}\right\} .
$$

\subsection{Interval Analysis}

In [I]RMOA method, the optimization parameters are modeled as punctual and interval variables. This subsection addresses the background on Interval Analysis necessary to understand how [I]RMOA deals with interval variables (further information can be found in $[15,16])$.

A real, closed and connected interval $[x]$ is defined as:

$$
[\underline{x}, \bar{x}]=\{x \in \mathbb{R} \mid \underline{x} \leq x \leq \bar{x}\},
$$

being $\underline{x}$ and $\bar{x}$, respectively, the lower and upper bounds of $[x]$. A generic real interval $[x]$, given by (5), is defined on the domain $\mathbb{R}$. The multi-dimensional real interval $[\mathbf{x}] \in \mathbb{R}^{n}$, named box or interval vector, is given by the Cartesian product of $n$ closed intervals:

$$
[\underline{\mathbf{x}}, \overline{\mathbf{x}}]=\left[x_{1}\right] \times\left[x_{2}\right] \times \cdots \times\left[x_{n}\right]
$$

where $\underline{\mathbf{x}}$ and $\overline{\mathbf{x}}$ represent, respectively, the lower and the upper bounds of $[\mathbf{x}]$.

Particularly, two properties of boxes are utilized by $[I]$ RMOA. The center $\mathbf{c}([\mathbf{x}])$ and the width $w([\mathbf{x}])$ of each box $[\mathbf{x}]$ are defined as:

$$
\begin{aligned}
& c_{i}([\mathbf{x}])=\left(\bar{x}_{i}+\underline{x}_{i}\right) / 2, \quad i=1, \ldots, n ; \\
& w([\mathbf{x}])=\max \left(\bar{x}_{i}-\underline{x}_{i}\right), \quad i \in\{1, \ldots, n\} .
\end{aligned}
$$

Using $\diamond$ to denote a binary operator that represents the four classical operations of the classic arithmetic,,$+- *$ and $/$, the interval arithmetic operations are defined as:

$$
[\mathbf{x}] \diamond[\mathbf{y}]=\{\mathbf{x} \diamond \mathbf{y} \mid \mathbf{x} \in[\mathbf{x}], \mathbf{y} \in[\mathbf{y}]\} .
$$

Formally, in the interval arithmetic, the four classical operations are applied directly to the bounds of the intervals likewise in (7) and (8). 
As it will be shown in Section 4, in order to guarantee (in a pessimistic and conservative way) that all uncertainties are preserved from being neglected, [I]RMOA utilizes the concept of inclusion function to estimate the objective functions for a generic box $[\mathbf{x}]$, whenever it is necessary. Given a function $\mathbf{f}: \mathbb{R}^{n} \rightarrow \mathbb{R}^{m}$, any function $[\mathbf{f}]: \mathbb{R}^{n} \rightarrow \mathbb{R}^{m}$ that satisfies the condition:

$$
\forall[\mathbf{x}] \in \mathbb{I} \mathbb{R}^{n}, \quad \mathbf{f}([\mathbf{x}]) \subset[\mathbf{f}]([\mathbf{x}])
$$

is considered an inclusion function for $\mathbf{f}$. However, as the quality of the final enclosure generated by [I]RMOA depends on the quality of the inclusion function, it is important to define high-quality inclusion functions for each objective function. For instance (see [15], p. 22), consider function $f(x)=x^{2}-x$ and two possible inclusion functions for $f(x):[f]([x])=[x]^{2}-[x]$ and $[f]^{\prime}([x])=\left([x]-\frac{1}{2}\right)^{2}-\frac{1}{4}$. When both inclusion functions are evaluated at the interval $[-1,3]$, we have:

$$
\begin{aligned}
& {[f]([-1,3])=[-1,3]^{2}-[-1,3]=[0,9]-[-1,3]=[-3,10] ;} \\
& {[f]^{\prime}([-1,3])=([-1,3]-0.50)^{2}-0.25=[-1.50,2.50]^{2}-0.25=[-0.25,6] .}
\end{aligned}
$$

As it can be seen, the interval provided by $[f]([x])$ is wider (or less precise) than the one provided by $[f]^{\prime}([x])$. Such difference in the precision of both resulting intervals is a consequence of the conservative and pessimistic nature of the Interval Analysis, which is reflected by the way the interval arithmetic operations are calculated. When the interval function involves more than one occurrence of the same interval variable, pessimistic intervals are constructed. Consequently, in order to obtain more precise results, it is interesting to minimize these occurrences in the inclusion function expression. References [17] and [18] provide, respectively, further information on the quality of inclusion functions and a discussion on the pessimism of inclusion functions.

In the robust approach described here, the dominance relation (2) is extended to deal with boxes. Thus, given the vector $\mathbf{u} \in \mathbb{R}^{m}$ and the boxes $[\mathbf{t}] \in \mathbb{R}^{m}$ and $[\mathbf{z}] \in \mathbb{R}^{m}$, the following relations are valid:

$$
\begin{aligned}
& \mathbf{u} \preceq[\mathbf{t}] \Leftrightarrow \mathbf{u} \preceq \underline{\mathbf{t}}, \\
& {[\mathbf{z}] \preceq[\mathbf{t}] \Leftrightarrow \overline{\mathbf{z}} \preceq \underline{\mathbf{t}} .}
\end{aligned}
$$

As it will be explained in Section 4, when [I]RMOA cannot conclude whether a box is dominated or not by other box, because of the uncertainty inherent to the boxes, the bisection operator described below is applied to reduce the imprecision of the boxes being compared. The regular bisection operator splits a box [x] into two boxes of the same size, given by:

$$
\begin{aligned}
& {[\mathbf{x}]_{1}=\left[x_{1}\right] \times \cdots \times\left[\underline{x}_{j}, \frac{\underline{x}_{j}+\bar{x}_{j}}{2}\right] \times \cdots \times\left[x_{n}\right],} \\
& {[\mathbf{x}]_{2}=\left[x_{1}\right] \times \cdots \times\left[\frac{\underline{x}_{j}+\bar{x}_{j}}{2}, \bar{x}_{j}\right] \times \cdots \times\left[x_{n}\right],}
\end{aligned}
$$

where $j$ is the index associated to the first component with maximum width, i.e., $j=\min \left\{i \mid w\left(\left[x_{i}\right]\right)=w([\mathbf{x}])\right\}$.

\section{Robust multi-objective optimization problem}

The traditional unconstrained multi-objective model (1) can be easily extended to include uncertainties. The robust version of (1) is supposed to incorporate all uncertainty factors identified in the analysis of the problem. Considering the design variables $\mathbf{x} \in \mathbf{X} \subseteq \mathbb{R}^{n}$ and the uncertainty parameter $\mathbf{p} \in \mathbf{P} \subseteq \mathbb{R}^{k}$, the new vector of objective functions is defined by $\mathbf{f}(\mathbf{x}, \mathbf{p}): \mathbb{R}^{n} \times \mathbb{R}^{k} \rightarrow \mathbb{R}^{m}$ and the original problem (1) can be rewritten as a robust unconstrained minimization problem:

$$
\min _{\mathbf{x} \in \mathbf{X}} \max _{\mathbf{p} \in \mathbf{P}} \mathbf{f}(\mathbf{x}, \mathbf{p}) \text {. }
$$

Solving (17) consists in finding the set of robust solutions $\mathbf{X}^{*}$, considering the influence of the uncertainties $\mathbf{p}$ :

$$
\mathbf{X}^{*}=\left\{\mathbf{x}^{*} \in \mathbf{X} \mid \nexists \mathbf{x} \in \mathbf{X}, \max _{\mathbf{p} \in \mathbf{P}} \mathbf{f}(\mathbf{x}, \mathbf{p}) \preceq \max _{\mathbf{p} \in \mathbf{P}} \mathbf{f}\left(\mathbf{x}^{*}, \mathbf{p}\right)\right\} .
$$

Given that $[\mathbf{Y}]=[\mathbf{f}](\mathbf{X}, \mathbf{P})$, the upper robust frontier $\mathbf{Y}^{+}$, the bottom robust frontier $\mathbf{Y}^{-}$and the robust Pareto frontier $\mathbf{Y}^{*}$ are, respectively, given by:

$$
\begin{aligned}
& \mathbf{Y}^{+}=\left\{\mathbf{y}^{+} \in[\mathbf{Y}] \mid \exists \mathbf{x} \in \mathbf{X}, \forall \mathbf{p} \in \mathbf{P}, \mathbf{f}(\mathbf{x}, \mathbf{p}) \preceq \mathbf{y}^{+}\right\}, \\
& \mathbf{Y}^{-}=\left\{\mathbf{y}^{-} \in[\mathbf{Y}] \mid \mathbf{y}^{-} \notin \mathbf{Y}^{+}\right\}, \\
& \mathbf{Y}^{*}=\left\{\mathbf{y}^{*} \in \mathbf{Y}^{+} \mid \nexists \mathbf{y} \in \mathbf{Y}^{+}, \mathbf{y} \preceq \mathbf{y}^{*}\right\} .
\end{aligned}
$$

These regions can be related as follows:

$$
[\mathbf{Y}]=\mathbf{Y}^{-} \cup \mathbf{Y}^{+}, \quad \mathbf{Y}^{*} \subseteq \mathbf{Y}^{+} .
$$




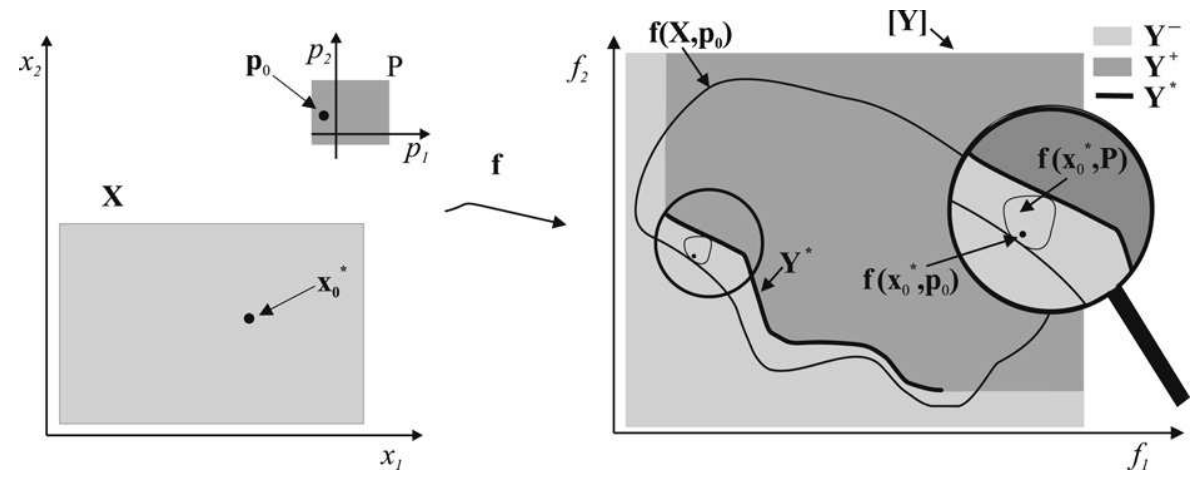

Fig. 1. Search and function spaces in a generic robust multi-objective problem.

It is worth mentioning that a generic robust multi-objective optimization problem can be seen as the maximization of the uncertainty effect on the objective functions, coupled with the subsequent minimization of all objective functions. Therefore, the robust image of each feasible point $\mathbf{x}$ corresponds to the vector $\mathbf{f}(\mathbf{x}, \mathbf{p})$ evaluated at point $\mathbf{x}$, considering the value of the parameter $\mathbf{p}$ that provides the highest value of $\mathbf{f}(\mathbf{x}, \mathbf{p})$.

The right side of Fig. 1 illustrates regions $\mathbf{Y}^{+}, \mathbf{Y}^{-}$and $\mathbf{Y}^{*}$, in the objective function space, being $\mathbf{f}\left(\mathbf{x}_{\mathbf{0}}^{*}, \mathbf{P}\right)$ the image of a specific robust solution $\mathbf{X}_{\mathbf{0}}^{*}$; $\mathbf{P}$ corresponds to the full uncertainty region and $\mathbf{X}$ is the full search region. Searching for the true robust Pareto frontier $\mathbf{Y}^{*}$ is a hard task. However, it is easier and it is also useful to compute an enclosure for $\mathbf{Y}^{*}$ as it will be shown in the next section.

\section{4. [I]RMOA}

Fig. 1 illustrates robust regions for a hypothetic case. [I]RMOA allows a discrete representation of regions $\mathbf{Y}^{+}$and $\mathbf{Y}^{-}$, which enclosure $\mathbf{Y}^{*}$. The mathematical representation of these robust regions, utilized by [I]RMOA is described next.

\subsection{Exclusion of convex regions in [I]RMOA}

[I]RMOA performs the exclusion of a specific type of convex region, named hypercone. The convex set $\mathbf{S}_{\mathbf{u}}^{+} \subseteq \mathbb{R}^{m}$ is an upper hypercone with vertex $\mathbf{u} \in \mathbb{R}^{m}$ defined by:

$$
\mathbf{S}_{\mathbf{u}}^{+}=\left\{\forall \mathbf{v} \in \mathbb{R}^{m} \mid \mathbf{u} \preceq \mathbf{v}\right\} .
$$

Similarly, the lower hypercone $\mathbf{S}_{\mathbf{u}}^{-} \subseteq \mathbb{R}^{m}$ is the set:

$$
\mathbf{S}_{\mathbf{u}}^{-}=\left\{\forall \mathbf{v} \in \mathbb{R}^{m} \mid \mathbf{v} \preceq \mathbf{u}\right\} .
$$

Considering $\mathbf{u} \in \mathbb{R}^{m}$ and $\mathbf{v} \in \mathbb{R}^{m}$, then the expressions below are equivalent:

$$
\mathbf{u} \preceq \mathbf{v} \Leftrightarrow \mathbf{S}_{\mathbf{u}}^{-} \subseteq \mathbf{S}_{\mathbf{v}}^{-} \Leftrightarrow \mathbf{S}_{\mathbf{u}}^{+} \supseteq \mathbf{S}_{\mathbf{v}}^{+} .
$$

[I]RMOA works on the basis of the exclusion of the convex hypercones $\mathbf{S}_{\mathbf{u}_{i}}^{-}$and $\mathbf{S}_{\mathbf{v}_{i}}^{+}$, which are respectively approximated by the sets $\mathbf{K}^{-}$and $\mathbf{K}^{+}$:

$$
\begin{aligned}
& \mathbf{K}^{-}=\bigcup_{i=1}^{n} \mathbf{S}_{\mathbf{u}_{i}}^{-}, \\
& \mathbf{K}^{+}=\bigcup_{i=1}^{m} \mathbf{S}_{\mathbf{v}_{i}}^{+},
\end{aligned}
$$

where $\left\{\mathbf{u}_{1}, \mathbf{u}_{2}, \ldots, \mathbf{u}_{n}\right\} \subset \mathbf{Y}^{-}$and $\left\{\mathbf{v}_{1}, \mathbf{v}_{2}, \ldots, \mathbf{v}_{m}\right\} \subset \mathbf{Y}^{+}$. The upper frontier of $\mathbf{K}^{-}$is given by:

$$
\partial \mathbf{K}^{-}=\left\{\mathbf{u} \in \mathbf{K}^{-} \mid \nexists \mathbf{u}^{\prime} \in \mathbf{K}^{-}, \mathbf{u} \preceq \mathbf{u}^{\prime}\right\} .
$$

Similarly, the bottom frontier of $\mathbf{K}^{+}$is given by:

$$
\partial \mathbf{K}^{+}=\left\{\mathbf{v} \in \mathbf{K}^{+} \mid \nexists \mathbf{v}^{\prime} \in \mathbf{K}^{+}, \mathbf{v}^{\prime} \preceq \mathbf{v}\right\} .
$$

Considering that $\backslash$ is the complement operator, the portions of $\mathbf{Y}^{-}$and $\mathbf{Y}^{+}$that are indirectly estimated are given by:

$$
\triangle \mathbf{K}^{-}=\left(\mathbf{Y}^{-} \backslash \mathbf{K}^{-}\right) \cup \partial \mathbf{K}^{-} \text {and } \triangle \mathbf{K}^{+}=\left(\mathbf{Y}^{+} \backslash \mathbf{K}^{+}\right) \cup \partial \mathbf{K}^{+} .
$$

The theorem presented below guarantees that the [I]RMOA wraps the image of robust solutions. 


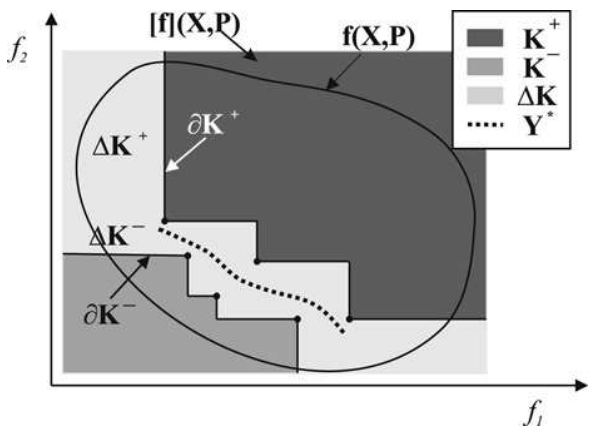

Fig. 2. Discrete approximation of the robust regions.

Theorem 1 (Enclosure of the Robust Pareto Frontier). Considering the precision to compute $\mathbf{K}^{-}$and $\mathbf{K}^{+}$, the following inclusion is always true

$$
\mathbf{Y}^{*} \subset \triangle \mathbf{K}^{-} \cup \triangle \mathbf{K}^{+} .
$$

Proof. From (22), we have that $[\mathbf{Y}]=\mathbf{Y}^{-} \cup \mathbf{Y}^{+}$. From (30), we have that: $\mathbf{Y}^{-}=\mathbf{K}^{-} \cup \Delta \mathbf{K}^{-}, \mathbf{K}^{-} \cap \Delta \mathbf{K}^{-}=\partial \mathbf{K}^{-}$and $\mathbf{Y}^{+}=\mathbf{K}^{+} \cup \triangle \mathbf{K}^{+}, \mathbf{K}^{+} \cap \triangle \mathbf{K}^{+}=\partial \mathbf{K}^{+}$. Substituting $\mathbf{Y}^{-}$and $\mathbf{Y}^{+}$results in

$$
[\mathbf{Y}]=\left(\mathbf{K}^{-} \cup \triangle \mathbf{K}^{-}\right) \cup\left(\mathbf{K}^{+} \cup \triangle \mathbf{K}^{+}\right) \text {. }
$$

But, $\mathbf{K}^{-} \cap \Delta \mathbf{K}^{-}=\partial \mathbf{K}^{-}$and $\mathbf{K}^{+} \cap \Delta \mathbf{K}^{+}=\partial \mathbf{K}^{+}$and thus, the extra occurrences of $\partial \mathbf{K}^{-}$and $\partial \mathbf{K}^{+}$can be removed. Then,

$$
\begin{aligned}
& {[\mathbf{Y}]=\left(\left(\mathbf{K}^{-} \backslash \partial \mathbf{K}^{-}\right) \cup \triangle \mathbf{K}^{-}\right) \cup\left(\left(\mathbf{K}^{+} \backslash \partial \mathbf{K}^{+}\right) \cup \triangle \mathbf{K}^{+}\right) ;} \\
& {[\mathbf{Y}]=\left(\left(\mathbf{K}^{-} \backslash \partial \mathbf{K}^{-}\right) \cup\left(\mathbf{K}^{+} \backslash \partial \mathbf{K}^{+}\right)\right) \cup\left(\triangle \mathbf{K}^{-} \cup \triangle \mathbf{K}^{+}\right) .}
\end{aligned}
$$

The expressions (21), (26) and (27) assert that $\mathbf{Y}^{*} \not \supset\left(\mathbf{K}^{-} \backslash \partial \mathbf{K}^{-}\right)$and $\mathbf{Y}^{*} \not \supset\left(\mathbf{K}^{+} \backslash \partial \mathbf{K}^{+}\right)$. Thus,

$$
\mathbf{Y}^{*} \subset \triangle \mathbf{K}, \quad \text { where } \triangle \mathbf{K}=\Delta \mathbf{K}^{-} \cup \triangle \mathbf{K}^{+} \text {. }
$$

Actually, (21) guarantees that $\mathbf{Y}^{*} \subset \Delta \mathbf{K}^{+}$. But $\triangle \mathbf{K}^{+}$alone cannot be directly enclosed. Fig. 2 illustrates the robust region representation discussed in this section.

\section{2. [I]RMOA method}

Basically, the search mechanism of [I]RMOA can be divided into two parts: (a) the main procedure; (b) the functions FPS (feasible point searcher) and CSC (computable sufficient conditions). Initially, in the main procedure of [I]RMOA, a grid is created, dividing the search region, into symmetrical pieces of $\varepsilon_{\#}$ size. The grid can be constructed in the search or in the function space, depending on the problem's dimension. When the problem involves too many optimization parameters, but few objective functions, the grid should be constructed in the function space to reduce the computational effort. Otherwise, it is advantageous to generate the grid in the search space. In this study, the grid is generated in the search space, without loss of generality. Such a grid contains points uniformly distributed along the search region $\mathbf{X}$. Each grid point $\mathbf{X}_{\mathbf{g}}$ is associated to an uncertainty parameter $\mathbf{p}$, which is generated at random, considering the predefined uncertainty region $\mathbf{P}$. All pairs $\left(\mathbf{x}_{\mathbf{g}}, \mathbf{p}\right)$, after objective function evaluation, are labeled as $\mathbf{y}_{\mathbf{g}}$ and stored in queue $\mathbf{Q}_{\mathbf{y}}$. Then, each $\mathbf{y}_{\mathbf{g}}$ is sequentially removed from $\mathbf{Q}_{\mathbf{y}}$ to verify whether it belongs to region $\mathbf{K}^{-}$or to region $\mathbf{K}^{+}$. This test is made based on the order operator (13), with the use of Interval Analysis to adequately deal with the uncertainty parameter $\mathbf{p}$. After classifying the whole queue into categories $\mathbf{K}^{-}$or $\mathbf{K}^{+},[I] R M O A$ has information to enclose $\mathbf{Y}^{*}$ since $\mathbf{Y}^{*} \subset \triangle \mathbf{K}$ and $\triangle \mathbf{K}=[\mathbf{Y}] \backslash\left(\mathbf{K}^{+} \cup \mathbf{K}^{-}\right)$.

The main procedure of [I]RMOA can be synthesized in terms of the steps below. For the sake of simplicity, the notation [.] is utilized to indicate the use of boxes or inclusion functions. It is worth emphasizing that the precision of the final enclosure depends on the input parameters $\varepsilon_{\#}$, which corresponds to the maximum width of the grid, and $\varepsilon_{[\mathbf{x}]}$, which corresponds to the minimum width of the box $[\mathbf{x}]$.

Step 1. Initialize $\mathbf{Q}_{\mathbf{y}}, \mathbf{Q}_{\mathbf{K}^{+}}, \mathbf{Q}_{\mathbf{K}^{-}}$as empty queues.

Step 2. Call function grid to generate the set of grid points $\mathbf{x}_{\mathbf{g}}$, uniformly distributed along the search region $\mathbf{X}$, and their respective uncertainty parameters $\mathbf{p}$, randomly sampled from the uncertainty region $\mathbf{P}$. Evaluate $\mathbf{y}_{\mathbf{g}}=\mathbf{f}\left(\mathbf{x}_{\mathrm{g}}, \mathbf{p}\right)$ for each pair $\left(\mathbf{x}_{\mathbf{g}}, \mathbf{p}\right)$, and insert each resulting $\mathbf{y}_{\mathbf{g}}$ in the end of $\mathbf{Q}_{\mathbf{y}}$.

Step 3. While $\mathbf{Q}_{\mathbf{y}}$ remains not empty, execute Steps 3.a, 3.b:

Step 3.a. Remove $\mathbf{y}_{\mathbf{g}}$ from the beginning of $\mathbf{Q}_{\mathbf{y}}$. 
Step 3.b. Verify if there is a point $\mathbf{v}$ in $\mathbf{Q}_{\mathbf{K}^{+}}$, that satisfies the condition $\mathbf{v} \preceq \mathbf{y}_{\mathbf{g}}$, and if there is a point $\mathbf{u}$ in $\mathbf{Q}_{\mathbf{K}^{-}}$, that satisfies the condition $\mathbf{y}_{\mathbf{g}} \preceq \mathbf{u}$. Iff none of these conditions is fulfilled, go to Step 4.

Step 4. Call functions FPS and CSC to verify if $\mathbf{y}_{\mathbf{g}}$ belongs to $\mathbf{K}^{+}$(and should be inserted into $\mathbf{Q}_{\mathbf{K}^{+}}$) or if $\mathbf{y}_{\mathbf{g}}$ belongs to $\mathbf{K}^{-}$(and should be inserted into $\mathbf{Q}_{\mathbf{K}^{-}}$).

Step 5. If $\mathbf{y}_{\mathbf{g}}$ belongs to $\mathbf{K}^{+}$, then delete in $\mathbf{Q}_{\mathbf{K}^{+}}$all elements $\mathbf{v}$ that satisfy the condition $\mathbf{y}_{\mathbf{g}} \preceq \mathbf{v}$ and insert $\mathbf{y}_{\mathbf{g}}$ in $\mathbf{Q}_{\mathbf{K}^{+}}$. Otherwise, delete in $\mathbf{Q}_{\mathbf{K}^{-}}$all elements $\mathbf{u}$ that satisfy the condition $\mathbf{u} \preceq \mathbf{y}_{\mathbf{g}}$ and insert $\mathbf{y}_{\mathbf{g}}$ in $\mathbf{Q}_{\mathbf{K}^{-}}$.

Step 6. If $\mathbf{Q}_{\mathbf{y}}$ is not empty, go to Step 3. Otherwise, go straight ahead.

Step 7. Compute $\mathbf{K}^{-}$, on the basis of (26), for each $\mathbf{u} \in \mathbf{Q}_{\mathbf{K}^{-}}$.

Step 8. Compute $\mathbf{K}^{+}$, on the basis of (27), for each $\mathbf{v} \in \mathbf{Q}_{\mathbf{K}^{+}}$.

Step 9. Return $\mathbf{K}^{-}$and $\mathbf{K}^{+}$.

FPS and CSC, proposed in [19], are utilized to verify if $\mathbf{y}_{\mathbf{g}}$ belongs to $\mathbf{K}^{-}$or to $\mathbf{K}^{+}$, taking into account the worst case scenario, characterized by the uncertainty parameter $[\mathbf{p}]$. While the current width of [p] does not allow CSC to precisely answer this question, CSC sequentially increases the precision of the inclusion function evaluation using the bisection operator to split [p]. On the other hand, when it is the current width of $\left[\mathbf{x}_{\mathbf{g}}\right]$ that does not allow CSC to precisely answer this question, in the FPS procedure, $\left[\mathbf{x}_{\mathbf{g}}\right]$ is bisected.

\section{Results}

Two types of tests are shown here. Type (a) is associated with tuning the [I]RMOA's input parameters to obtain a satisfactory enclosure of the robust Pareto frontier. In order to verify the adequacy of the enclosure, it is compared with the true robust Pareto frontier for the worst case scenario, defined by a specific uncertainty region $\mathbf{P}$. It is interesting to note that the worst case scenario characterized by $\mathbf{P}$ may change if a different uncertainty region is specified. However, after tuning the precision parameters, [I]RMOA can construct an enclosure of the robust Pareto frontier for other worst case scenario. Type (b) is associated with the validation of an evolutionary algorithm with the use of [I]RMOA. In these tests, the robust Pareto frontier obtained by a stochastic search algorithm is acceptable, when it is inside the wrapper provided by [I]RMOA. The elitist multi-objective genetic algorithm from [7] (used to solve a robust multi-objective design optimization problem) was chosen to perform the Type (b) tests. In this stochastic framework, we redefined the robust problem (17) as follows:

$$
\min _{\mathbf{x} \in \mathbf{X}} \max _{\mathbf{p}^{\prime} \in \mathbf{P}^{\prime}} \mathbf{f}\left(\mathbf{x}, \mathbf{p}^{\prime}\right),
$$

where $\mathbf{P}^{\prime}$ is a discrete set with samples randomly chosen from $\mathbf{P}$. Thus, the validation consists in searching a suitable size of samples $\mathbf{P}^{\prime}$ that can be utilized by the stochastic search algorithm to adequately represent the robust Pareto front.

Two nonrobust multi-objective optimization problems, ZDT3 [20] and FON [21], were stated as robust unconstrained minimization problems:

$$
\begin{aligned}
& \text { ZDT3 }^{\prime} \begin{cases}\min _{\mathbf{x} \in[\mathbf{0}, 1]} \max _{p \in[-0.1,0.1]} & f_{1}(\mathbf{x})=x_{1} ; \\
& f_{2}(\mathbf{x})=h^{\prime}(\mathbf{x}, p) \times h\left(f_{1}, h^{\prime}, p\right) . \\
\text { where: } & h^{\prime}(\mathbf{x}, p)=1+\frac{9}{n-1}\left(\left(\sum_{i=2}^{n} x_{i}\right)+p\right), \\
& h\left(f_{1}, h^{\prime}, p\right)=1-\sqrt{f_{1} / h^{\prime}}-\left(f_{1} / h^{\prime}\right) \sin \left(10 \pi f_{1}\right) .\end{cases} \\
& F O N^{\prime}=\left\{\begin{aligned}
\min _{\mathbf{x} \in[-\mathbf{4}, \mathbf{4}]} \max _{p \in[1.1,1.3]} f_{1}(\mathbf{x}, p)=1-\mathrm{e}^{-\sum_{i=1}^{n}\left(x_{i}-\frac{p}{\sqrt{n}}\right)^{2}} \\
f_{2}(\mathbf{x}, p)=1-\mathrm{e}^{-\sum_{i=1}^{n}\left(x_{i}+\frac{p}{\sqrt{n}}\right)^{2}}
\end{aligned}\right.
\end{aligned}
$$

where $n$ is the size of the vector of design variables. In both examples, $n$ was set as 2 . The robust Pareto frontier of ZDT3' is nonconvex and discontinuous and the robust Pareto frontier of FON' is nonconvex. Whereas FON' has a sharp objective inclusion function, the objective inclusion function of ZDT3' can be considered highly conservative as it involves multiple occurrences of variable $x_{1}$. The uncertainty parameter $\mathbf{p}$, inserted into ZDT3', can be seen as the imprecision intrinsic to the measure of the objective functions. On the other hand, the uncertainty parameter p, inserted into FON', represents a parametric uncertainty. Fig. 3 shows the feasible region of both ZDT3' and FON', mapped to the objective function space. In Fig. 3, $\mathbf{Y}_{\mathbf{d}}^{*}$ is the discrete representation of robust Pareto frontier directly estimated with the use of (32) and (33).

In Type (a) tests, when ZTD3' was considered, the [I]RMOA input parameters were set with the following values: $\varepsilon_{\#} \in\{0.01,0.02, \ldots, 0.1\}$ and $\varepsilon_{[\mathbf{x}]} \in\{0.01: 0.01: 0.1\}$. On the other hand, $\varepsilon_{\#} \in\{0.05,0.10,0.15, \ldots, 1.0\}$ and $\varepsilon_{[\mathbf{x}]} \in\{0.2,0.3, \ldots, 1.0\}$ for FON'. The inclusion functions were obtained by direct substitution of the variables by their 


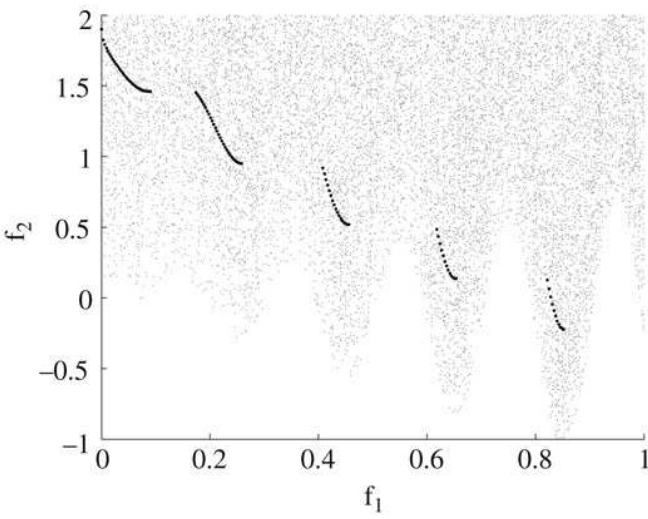

(a) $\mathrm{ZDT}^{\prime} ; n=2$.

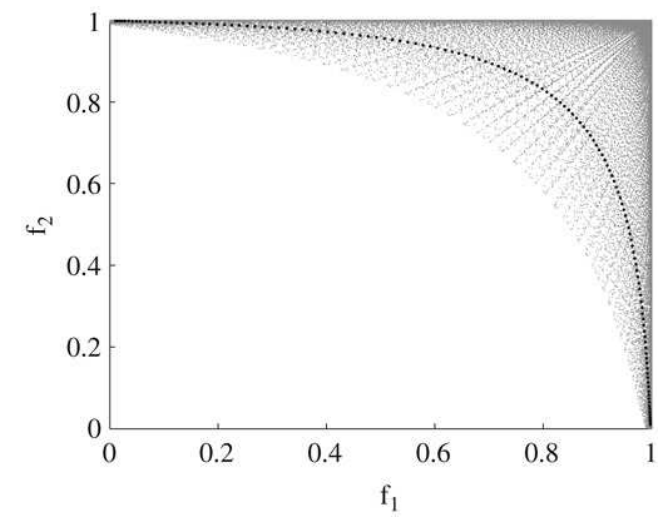

(b) $\mathrm{FON}^{\prime} ; n=2$.

Fig. 3. Robust objective space. In gray, some samples of $\mathbf{f}(\mathbf{X}, \mathbf{P})$; in black the discrete representation of the robust Pareto frontier $\mathbf{Y}_{\mathbf{d}}^{*}$.

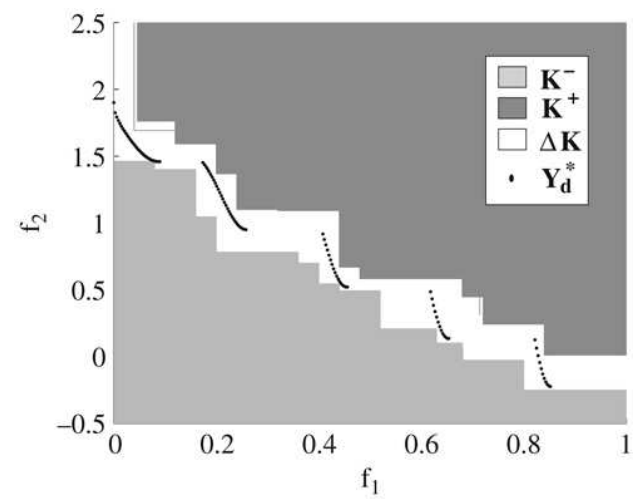

(a) $\operatorname{ZDT3}^{\prime}\left(\varepsilon_{[\mathbf{x}]}=0.01, \varepsilon_{\mathbf{x} \#}=0.04\right)$

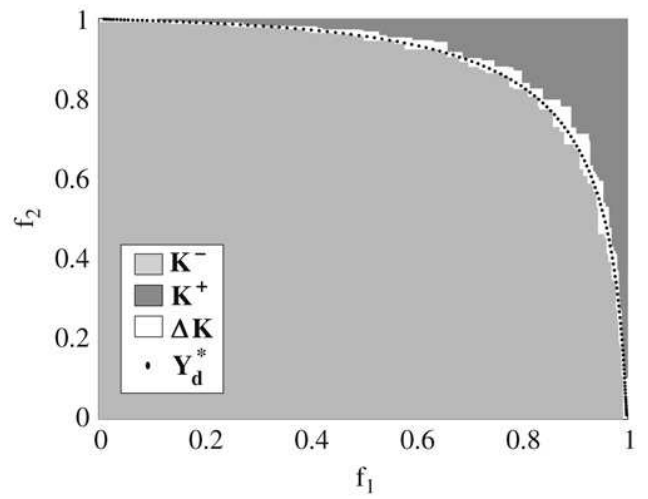

(b) $\operatorname{FON}^{\prime}\left(\varepsilon_{[\mathbf{x}]}=0.2, \varepsilon_{\mathbf{x} \#}=0.05\right)$.

Fig. 4. Enclosure provided by [I]RMOA for ZTD3' and FON'.

interval counterparts in (32) and (33). The best results related to Type (a) tests are shown in Fig. 4. In order to construct the enclosures shown in Fig. 4, [I]RMOA required around $10^{7}$ objective function evaluations for ZDT3' and around $10^{6}$ objective function evaluations for FON'. As it can be seen, the enclosure of ZDT3' is less precise than the enclosure of FON'. It is a consequence of the fact that the quality of the objective inclusion function of ZDT3' is lower than the objective inclusion function of FON' (due to the multiple occurrences of the variable $x_{1}$ ).

In Type (b) tests, the values of the input parameters associated to the best results were used to validate the results obtained by the multi-objective genetic algorithm. It is interesting to mention that the stochastic search algorithm was executed 10 times (with a population of 50 individuals and with 40 generations) for ten different sample sizes of $\mathbf{P}^{\prime}$ $(5,10, \ldots, 50$ samples). The most interesting results are exposed in Fig. 5.

Each size of $\mathbf{P}^{\prime}$ is associated with a robust Pareto frontier. The minimal acceptable size was the one with which the genetic algorithm could meet a robust Pareto frontier inside the wrapper generated by [I]RMOA. In these simple examples, 50 samples were the minimum acceptable size. It must be clear that the points met by the genetic algorithm located in $\mathbf{K}^{+}$region certainly do not belong to the robust Pareto set, for a simple reason: [I]RMOA may overestimates the objective inclusion functions.

\section{Conclusions}

Since [I]RMOA provides acceptable enclosures of the robust Pareto frontier for a given uncertainty region P, [I]RMOA can be utilized to validate other robust multi-objective optimization algorithms, which deal with the worst case scenario. This characteristic may be very useful, specially when the shape of the robust Pareto frontier is unknown. [I]RMOA has some other particular characteristics that should be strengthened. It is a versatile tool, as it can be applied to find an enclosure of the robust Pareto frontier for problems with different features, depending only on the availability of objective inclusion functions to model the problem. [I]RMOA has few parameters, which are related to the precision of its results. [I]RMOA may require high processing time. However, the computational effort can be reduced with the use of other interval and noninterval methodologies. This is our next research direction. 


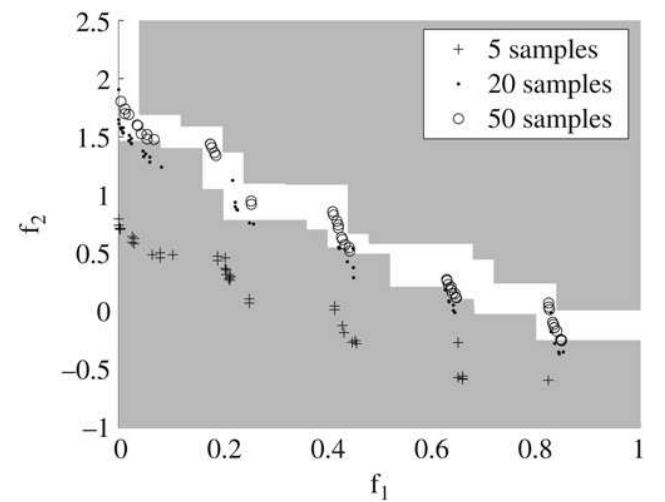

(a) $\operatorname{ZDT3}\left(\varepsilon_{[\mathbf{x}]}=0.01, \varepsilon_{\mathbf{x} \#}=0.04\right)$.

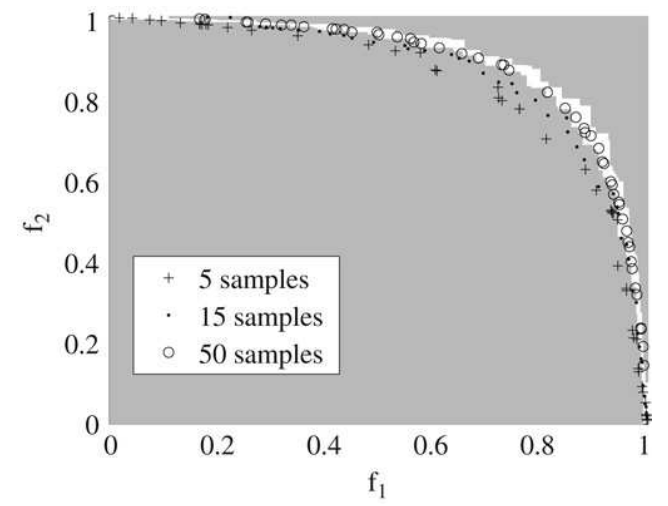

(b) $\operatorname{FON}^{\prime}\left(\varepsilon_{[\mathbf{x}]}=0.2, \varepsilon_{\mathbf{x} \#}=0.05\right)$.

Fig. 5. Validation of a genetic algorithm. The small samplings produce the worst representations to robust Pareto front.

\section{Acknowledgements}

In Brazil, this work was supported by Fapemig (grant TEC 00140/07), CNPq (grant 142523/2007-0) and PUCMinas; In France, by ENSIETA and UBO.

\section{References}

[1] H.G. Beyer, B. Sendhoff, Robust optimization - a comprehensive survey, Computer Methods in Applied Mechanics and Engineering 196 (2007) 3190-3218.

[2] X. Du, W. Chen, Towards a better understanding of modeling feasibility robustness in engineering design, 1999.

[3] Y. Jin, J. Branke, Evolutionary optimization in uncertain environments-a survey, IEEE Transactions on Evolutionary Computation 9 (2005) $303-317$.

[4] A. Ben-Tal, A. Nemirovski, Robust optimization - methodology and applications, Mathematical Programming (Series B) 92 (2002) 453-480.

[5] D. Bertsimas, M. Sim, The price of robustness, Operations Research 52 (1) (2004) 35-53.

[6] L. El Ghaoui, F. Oustry, H. Lebret, Robust solutions to uncertain semidefinite programs, SIAM Journal of Optimization 9 (1) (1998) 33-52.

[7] G.L. Soares, R.L.S. Adriano, C.A. Maia, L. Jaulin, J.A. Vasconcelos, Robust multi-objective team 22 problem: A case study of uncertainties in design optimization, IEEE Transactions on Magnetics 45 (2009) 1028-1031.

[8] D.S. Lee, L.F. Gonzalez, J. Periaux, K. Srinivas, Robust design optimisation using multi-objective evolutionary algorithms, Computers \& Fluids 37 (5) (2008) 565-583. Special Issue Dedicated to Professor M.M. Hafez on the Occasion of his 60th Birthday.

[9] P. Kouvelis, G. Yu, Robust Discrete Optimization and Its Applications, Kluwer Academic Publishers, 1997.

[10] C. Jiang, X. Han, G.R. Liu, G.P. Liu, A nonlinear interval number programming method for uncertain optimization problems, European Journal of Operational Research 188 (1) (2008) 1-13.

[11] G.C. Calafiore, M.C. Campi, The scenario approach to robust control design, IEEE Transactions on Automatic Control 51 (5) (2006) $742-753$.

[12] N.V. Sahinidis, Optimization under uncertainty: State-of-the-art and opportunities, Computers and Chemical Engineering 28 (2004) $971-983$.

[13] R.T. Marler, J.S. Arora, Survey of multi-objective optimization methods for engineering, Structural and Multidisciplinary Optimization 26 (2004) 369-395.

[14] K. Miettinen, Nonlinear Multiobjective Optimization, in: International Series in Operations Research and Management Science, vol. 12, Kluwer Academic Publishers, Dordrecht, 1999.

[15] L. Jaulin, M. Kieffer, O. Didrit, E. Walter, Applied Interval Analysis, Springer, 2001.

[16] E.R. Hansen, G.W. Walster, Global Optimization Using Interval Analysis, 2nd ed., Marcel Dekker Inc., 2004.

[17] H. Ratschek, J. Rokne, Computer Methods for the Range of Functions, Halsted Press, 1984.

18] G. Chabert, L. Jaulin, Computing the pessimism of inclusion functions, Reliable Computing 13 (6) (2007) 489-504.

[19] L. Jaulin, E. Walter, Guaranteed tuning, with application to robust control and motion planning, Automatica 32 (8) (1996) $1217-1221$.

[20] E. Zitzler, K. Deb, L. Thiele, Comparison of multiobjective evolutionary algorithms: Empirical results, Evolutionary Computation Journal 8 (2) (2000) $125-148$.

[21] C.M. Fonseca, P.J. Fleming, An overview of evolutionary algorithms in multi-objective optimization, Evolutionary Computation Journal 3 (1995) 1-16. 